\title{
An Integrated Architecture for Corrosion Monitoring and Testing, Data mining, Modeling and Diagnostics/Prognostics
}

\author{
Honglei $\mathrm{Li}^{1}$, Margaret R Garvan ${ }^{2}$, Jiaming Li ${ }^{3}$, Javier Echauz ${ }^{4}$, George J. Vachtsevanos ${ }^{5}$, Douglas W. Brown ${ }^{6}$, Richard J. \\ Connolly $^{7}$, Frank Zahiri ${ }^{8}$ \\ 1,2,3,5 Department of Electrical and Computer Engineering, Georgia Institute of Technology, Atlanta, GA, USA \\ gjv@ece.gatech.edu \\ mgarvan3@gatech.edu \\ jli339@gatech.edu \\ gjv@ece.gatech.edu \\ ${ }^{4}$ JE Research, Inc., Alpharetta, GA, USA \\ echauz@ieee.org \\ ${ }^{6,7}$ Analatom, Inc., Santa Clara, CA, USA \\ Doug.Brown@analatom.com \\ Richard.Connolly@analatom.com \\ ${ }^{8}$ Warner Robins Air Force Base, Warner Robins, GA, USA \\ Feraidoon.zahiri@us.af.mil
}

\begin{abstract}
It has been established that corrosion is one of the most important factors causing deterioration and decreased performance and reliability in critical aerospace and industrial systems. Corrosion monitoring, detection, and quantification are recognized as key enabling technologies to reduce the impact of corrosion on the integrity of aircraft and industrial assets. Accurate and reliable detection of corrosion initiation and propagation, with specified false alarm rates, requires novel tools and methods, including verifiable simulation and modeling methods. This paper reports an experimental investigation of the detection and quantification of pitting corrosion on aluminum alloy panels using 3D surface metrology methods and image processing techniques. Panel surfaces were evaluated by laser microscopy and stylus-based profilometry to characterize global and local surface features. Promising imaging and texture features were extracted and compared between coated and uncoated aluminum panels at different exposure times under accelerated corrosion conditions. Image processing, information processing, and data mining techniques were utilized to evaluate the presence and extent of pitting corrosion. A new modeling framework for corrosion stages is
\end{abstract}

Honglei Li et al. This is an open-access article distributed under the terms of the Creative Commons Attribution 3.0 United States License, which permits unrestricted use, distribution, and reproduction in any medium, provided the original author and source are credited. introduced that emphasizes the representation of pitting corrosion and ultimately the crack formation process. Detection and prediction of the evolution of corrosion stages relies on data, a particle filtering method, and the corrosion propagation model. Results from these experimental studies demonstrate the efficacy of this proposed methodology.

\section{INTRODUCTION}

Corrosion is known to cause the loss of billions of dollars every year in structural integrity deterioration, leading to decreased performance and reliability of military and civil engineering assets. In aerospace industries for example, aluminum alloys used in aircraft fuselage lap-joints are subjected to crevice corrosion damage, especially localized corrosion pillowing, which may cause unanticipated and catastrophic system failures (Wallace \& Hoeppner, 1985) (Wei, Liao, \& Gao, 1998). Corrosion states take various forms starting with microstructure corrosion and ending with stress induced cracking (Hoeppner, Chandrasekaran, \& Taylor, 1999) (Kawai \& Kasai, 1985) (Lindley, Mcintyre, \& Trant, 1982). Generally, corrosion starts in the form of pitting, due to the presence of a surface contaminant or material heterogeneity. Facilitation of this process occurs by the interaction of the corrosive environment and cyclic loading, resulting in fatigue crack initiation across pitted areas that further grows and leads to accelerated structural failure (Pidaparti, 2007). In order to effectively conduct structural corrosion health assessment, it is crucial to 
understand how corrosion initiates from the microstructure to the component level through corrosion modeling, and how structural corrosion behaviors change as a result of varied environmental stresses by incorporating these factors into the modeling process. In addition, facilitated by understanding and modeling of corrosion growth and associated processes, continuous monitoring, detection, localization, and quantification of corrosion, and further, prediction of corrosion damage growth in complex structures over large, partially accessible areas are of growing interest in the aerospace industries. The aforementioned technical challenges of corrosion assessment and rising cost of existing corrosion-related maintenance call for both the exploration of new and innovative structural corrosion health assessment techniques that integrate robust corrosion testing and monitoring, data mining, corrosion detection, and prediction of corrosion damage growth, with intelligent reasoning paradigms that capture historical data, expert opinion, and adaptation strategies to associate current evidence with past cases obtained fleet-wide for similar system components.

Pitting corrosion represents the initial phase of corrosion, and is one of the most prevalent forms of localized corrosion, a dangerous phenomenon because of its rapid damage growth rate, and the difficulty associated with detecting and predicting its evolution. The pitting attack is highly localized and is usually in the form of holes that can penetrate inwards extremely rapidly and ultimately damage the structure by either perforating the material or developing into cracking due to stress corrosion (Rao \& Rao, 2004). Thus it is essential to insure the integrity and operational safety of the critical asset by condition-based monitoring, early detection, interpretation and prediction of pitting attack. Many research efforts have been reported in the past addressing this serious issue (Frankel, 1998) (Huang \& Frankel, 2006) (SzklarskaSmialowska, 1999) (Pereira, Silva, Acciari, Codaro, \& Hein, 2012). Traditionally, conventional ultrasonic and eddy current techniques have been used to precisely measure the reduction in thickness in aircraft structures. However, scanning may become impractical when inspection area is inaccessible. To address this a number of research techniques have been developed, including guided wave tomography to screen large areas of complex structure for corrosion detection, localization (Clark, 2009) and defect depth mapping (Belanger, Cawley, \& Simonetti, 2010). However, due to the nature of guided ultrasonic waves, this technique is vulnerable to environmental changes, especially temperature variation and surface wetness ( $\mathrm{Li}$, et al., 2012). The precision of corrosion defect depth reconstruction is restricted by sensor network layout and structure complexity, which limits the scope of field applications. Thus, wellrecognized global corrosion measurements, such as material weight loss and wall thickness reduction, cannot offer an appropriate and trustworthy way to interpret pitting corrosion due to its highly localized nature.
Advanced corrosion health assessment systems require comprehensive quantitative information, which can be categorized into a variety of feature groups, such as corrosion morphology, texture, and location. Implementation of advanced health assessment systems will require the exploration of new testing methods and data fusion methods from multiple testing techniques. Forsyth and Komorowski (Forsyth \& Komorwoski, 2000) discussed data fusion techniques to combine the information from multiple NDE techniques into an integrated form for structural modeling. Several other studies have looked into different sensing technologies for corrosion health monitoring, including the use of a micro-linear polarization resistance ( $\mu L P R)$ sensor (Brown, Darr, Morse, \& Laskowski, 2012) (Brown, et al., 2014) and fiber optic sensors (McAdam, Newman, McKenzie, Davis, \& Hinton, 2005). The existing research focused on a combination of surface metrology and image processing is very limited. In parallel to the current corrosion sensing technology, there have been a number of corrosion modeling studies attempting to numerically capture the processes of pitting corrosion initiation, pitting evolvement, pitting to cracking transition, and crack growth to fracture at the molecular level. Currently there is not a widely accepted quantitative model to take into consideration of the effect of stress factors (e.g. salinity, temperature, pressure), although the effects of the above-mentioned stress factors have been widely discussed.

The remainder of this paper is organized by the following: Section 2 outlines the PHM architecture followed in this study; Section 3 identifies the corrosion modeling-based / application specific features and feature extraction routines used in the development of the prognostic/diagnostic models; Section 4 outlines the experimental materials and methods followed in this study; Section 5 presents the modeling results; and finally Section 6 summarizes the findings of the study, lessons learned, and future work.

\section{PHM ARCHITECTURE}

Fault detection and prediction entails a series of functions. Starting with the monitoring apparatus, data/image collection and processing, corrosion modeling, detection, prediction, and, finally, assessment of the potential impact of corrosion on the operational integrity of an asset. The architecture is set as a decision support system providing advisories to the operator/maintainer as to the health status of critical aircraft component(s) subjected to corrosion and in need of corrective action. The sensing/modeling and diagnostic/prognostic functions are coupled with a novel reasoning paradigm called Dynamic Case Based Reasoning (DCBR) that houses a case library composed of past documented cases detailing the impact of cracking on the integrity of platform components/systems. The DCBR is supported by cognitive routines for learning and adaptation so that new evidence is compared with stored cases and those occurring for the first 


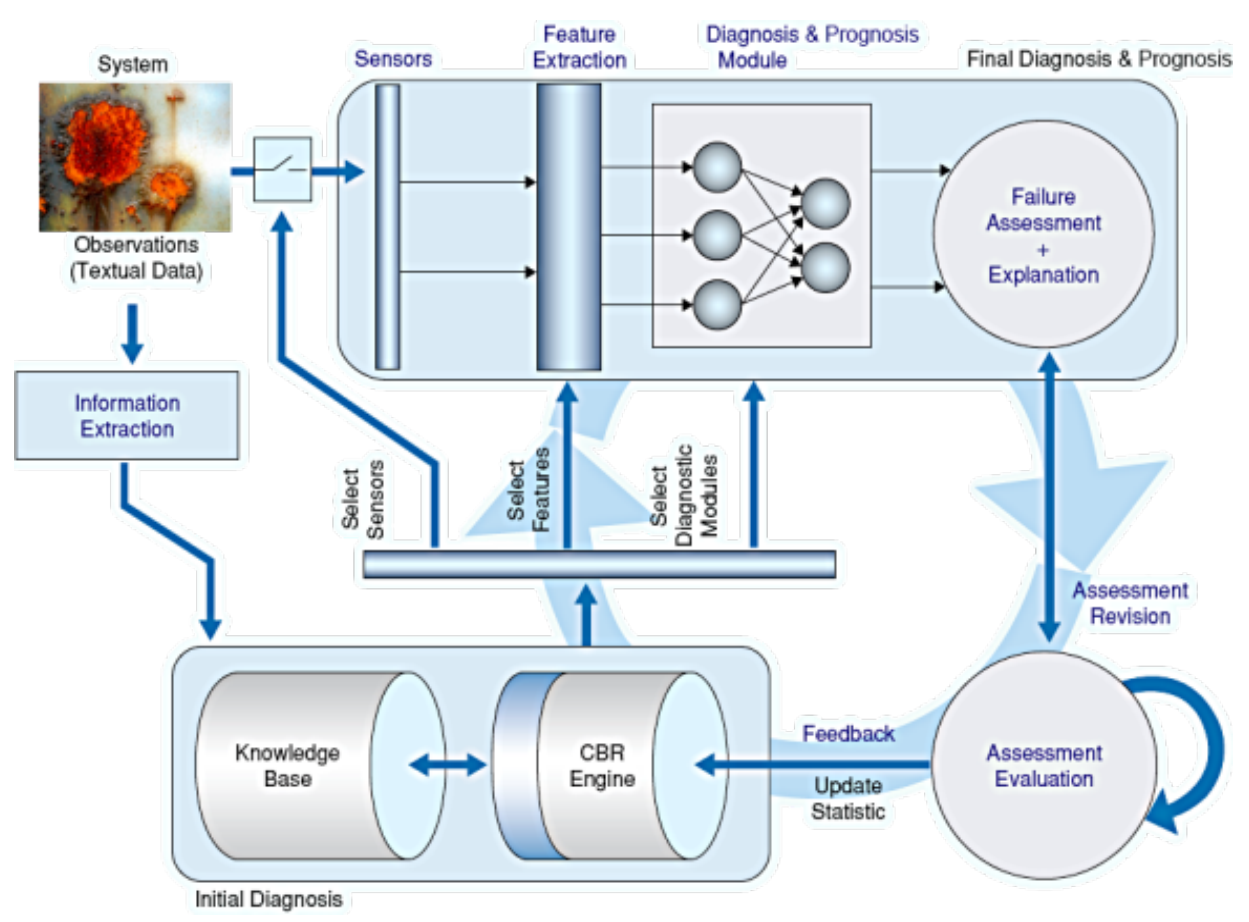

Figure 1. DCBR Architecture for integrated crack diagnosis, prognosis and maintenance.

time are "learned" by the reasoner. Finally, a corrosion severity index is defined to assist in assessing the impact of corrosion on the asset's structure integrity.

Figure 1 shows a conceptual schematic of the major modules of the corrosion processing and detection/prediction PHM architecture. The architecture combines a model-based and data-driven methodology taking advantage of experimental data, corrosion progression models, and an estimation method called particle filtering in order to detect the early initiation of corrosion. It is accompanied by performance metrics for detection confidence, false alarm rate, and prediction accuracy/precision (López De La Cruz, Lindelauf, Koene, \& Gutiérrez, 2007). In this architecture, the most important components supporting the implementation of the algorithm are feature extraction, fault diagnosis, and failure prognosis.

\subsection{Feature Selection, Extraction, and Identification}

Features form the foundation for the fault/corrosion detection and interpretation scheme. Feature selection, extraction and identification processes are optimized to extract only the information that is maximally correlated with the actual corrosion state. Features used in the development of fault diagnostic and prognostic models are discussed in more detail in Section 3.

\subsection{Fault diagnosis}

The Fault Detection and Identification (FDI) procedure may be interpreted as the fusion and utilization of the information present in a feature vector (observations) with the objective of determining the operational condition (state) of a system and the causes for deviations from particularly desired behavioral patterns (McAdam, Newman, McKenzie, Davis, \& Hinton, 2005).

A fault diagnosis procedure involves the tasks of fault detection and identification (assessment of the severity of the fault). In this sense, the proposed particle-filter-based diagnostic framework aims to accomplish these tasks, under general assumptions of non-Gaussian noise structures and nonlinearities in process dynamic models, using a reduced particle population to represent the state probability density function (PDF) (Straub, 2004). The particle filter-based module builds on the nonlinear dynamic state model,

$$
\left\{\begin{aligned}
x_{d}(t+1) & =f_{b}\left(x_{d}(t), n(t)\right), \\
x_{c}(t+1) & =f_{t}\left(x_{d}(t), x_{c}(t), w(t)\right), \\
f_{p}(t) & =h_{t}\left(x_{d}(t), x_{c}(t), v(t)\right),
\end{aligned}\right.
$$

where $f_{b}, f_{t}$ and $h_{t}$ are non-linear mappings, $x_{d}(t)$ is a collection of Boolean states associated with the presence of a particular operating condition in the system (normal operation, fault type $\# 1$, \#2, etc.), $x_{c}(t)$ is a set of continuousvalued states that describe the evolution of the system given those operating conditions, $f_{p}(t)$ is a feature measurement, $w(t)$ and $v(t)$ are non-Gaussian distributions that characterize the process and feature noise signals respectively. At any given instant in time, this framework provides an estimate of the probability masses associated 
with each fault mode, as well as a PDF estimate for meaningful physical variables in the system. The FDI module generates proper fault alarms and as well as the statistical confidence of the detection routine. Performance metrics are translated into acceptable margins for the type I (false positives) errors and type II errors (false negatives) in the detection routine. The algorithm itself will indicate when the type II error has decreased to the desired level.

\subsection{Failure Prognosis}

Prognosis is activated when a fault is detected. For the same fault mode, the propagation of the fault follows the same physical law. The prognostic framework takes advantage of a nonlinear process model, a Bayesian estimation method using particle filtering and real-time measurements. Prognosis is achieved by performing two sequential steps, prediction and filtering. Prediction uses the knowledge of the previous state estimate and the process model to generate the a priori state PDF for the next time instant:

$p\left(x_{0: t} \mid y_{1: t-1}\right)=\int p\left(x_{t} \mid x_{t-1}\right) p\left(x_{0: t-1} \mid y_{1: t-1}\right) d x_{0: t-1}$.

Unfortunately, this expression does not have an analytical solution in most cases. Instead, Sequential Monte Carlo (SMC) algorithms or particle filters are used to numerically solve this equation in real-time through the use of efficient sampling strategies. Particle filtering approximates the state PDF using samples or "particles" having associated discrete probability masses ("weights") as,

$$
p\left(x_{0: t} \mid y_{1: t}\right) \approx \widetilde{w}_{t}\left(x_{0: t}^{i}\right) \cdot \delta\left(x_{0: t}-x_{0: t}^{i}\right) d x_{0: t-1},
$$

where $x_{0: t}^{i}$ is the state trajectory and $y_{1: t}$ are measurements up to time $t$. The implementation of this algorithm, the Sequential Importance Re-sampling (SIR) particle filter, updates the weights using the likelihood of $y_{t}$ as,

$$
w_{t}=w_{t-1} \cdot p\left(y_{t} \mid x_{t}\right)
$$

Long-term predictions are used to estimate the probability of failure in a system given a hazard zone that is defined via a PDF with lower and upper bounds for the domain of the random variable, denoted as $H_{l b}$ and $H_{u p}$, respectively. The probability of failure at any future time instant is estimated by combining both the weights $w_{t+k}^{(i)}$ of predicted trajectories and specifications for the hazard zone through the application of the Law of Total Probabilities. The resulting Remaining Useful Life (RUL) PDF, where $t_{R U L}$ refers to RUL, provides the basis for the generation of confidence intervals and expectations for prognosis,

$$
\hat{p}_{t_{R U L}}=\sum_{i=1}^{n} p\left(\text { Failure } \mid X=\hat{x}_{t_{R U L}}^{(i)}, H_{l b}, H_{u p}\right) \cdot w_{R U L}^{(i)} .
$$

\subsection{Case Based Reasoning (CBR)}

CBR constitutes the main system level reasoning paradigm of the architecture and incorporates essential elements of a learning strategy. CBR was founded on the belief that human memory is episodic in nature. This episodic memory, which comprises human knowledge, is accumulated from past experience. Each memory episode is contributed by a single past situation or event. Faced with a new problem, a human often relates the problem to one or more memory episodes and composes a solution from these episodes. CBR is a computer program to simulate this human recognition process and has been applied to a variety of process operation support systems. The CBR application domain usually involves problem solving, i.e. identify similar cases for better understanding, assessing, and/or comparing with the current situation.

\section{Features ANd Modeling}

There are several characterization features to physically quantify the pitting corrosion attack, e.g. corroded area percentage, average pit depth measurement, maximum pit depth measurement, pitting density (pits/ $\mathrm{mm}^{2}$ ), and remaining wall thickness due to pitting. In addition, imageprocessing techniques can be used to extract morphological and texture features to facilitate pitting corrosion interpretation.

\subsection{Gray Level Co-occurrence Matrix (GLCM)}

2D imaging texture features, such as contrast, correlation, energy and homogeneity, summarized in Table 1 , are calculated using the normalized GLCM denoted as $p(i, j)$, where $i$ and $j$ correspond to rows and columns of the GLCM accordingly. The $(i, j)$ indices correspond to how often a pixel with value $i$ occurs horizontally adjacent to a pixel with value $j$ in image $I$. The contrast returns a measure of the intensity contrast between a pixel and its neighbor over the whole image. For a constant image, the contrast is 0 . The correlation returns a measure ranging between -1 and 1 that represents how correlated a pixel is to its neighbor over the entire image. The energy is calculated as the sum of the squared elements in the GLCM. For a constant image, the energy is 1 . Finally, the homogeneity is a measure of the closeness of the distribution of elements in the GLCM to its diagonal.

\subsection{Morphological Features}

Morphological features can be extracted from 2D pitting images to characterize the shape of the pitting attacked surface area. Features such as roundness, solidity, eccentricity, major axis length and minor axis length are calculated as shown in Table 2. For roundness, $A$ represents the area of the region and $p$ is the perimeter of the region. Solididy is defined as the ratio of the area of the region to the area of the convex hull region, represented by ConvexArea. 
For an ellipse defined by $(x / a)^{2}+(y / b)^{2}=1$, the eccentricity, major axis length, $L_{\text {Major }}$, and minor axis length, $L_{\text {Minor }}$ is computed as shown in Table 2.

\subsection{Surface Roughness Features}

Surface roughness is a measure of the texture of a surface. It is quantified by the vertical deviations $Z(x, y)$ of a real surface from its ideal form. If these deviations are large, the surface is rough; if they are small the surface is smooth. Roughness is typically considered to be the high frequency, short wavelength component of a measured surface. The 3D surface roughness features are listed in Table 3.

\subsection{Additional Geometric Features}

Other pit characterization features include the corroded area geometric features, 2D pit profile features, and 3D pit profile features.

\subsection{Feature Selection Performance Metrics}

After a sufficient number of features are extracted, feature selection can be conducted to determine the smallest subset of features that satisfies given performance criteria. Performance metrics such as correlation coefficient and Fisher discriminant ratio (FDR) can be applied to assess the feature quality. Optimization and principle component analysis (PCA) tools can be used for this purpose. Then a list of "best" features can be selected based on the feature performance. Here we use correlation coefficient and FDR to gauge the image features, as expressed in Table 4, where, $X$ and $Y$ are two random variables with expected values $\mu_{X}$ and $\mu_{Y}$ and standard deviations $\sigma_{X}$ and $\sigma_{Y}$.

\subsection{Corrosion Modeling}

A simplified model for corrosion growth presented by (Straub, 2004) predicts the corrosion depth from the combined contribution of uniform corrosion (surface loss) and localized corrosion (e.g. pitting \& crevice). However, no practicable framework currently exists for detailed spatial information (in 1-, 2-, or 3-D). Thus the focus turns to the maximum depth $d_{\max }(t)$ over a region (e.g. deepest pit in a whole pipe or panel), whose statistics can be treated with Gumbel or Weibull extreme value distributions. Data from atmospheric uniform corrosion support use of a power growth over time:

$$
d(t)=a t^{b},
$$

where $a$ and $b$ are coefficients dependent on temperature, relative humidity $(\mathrm{RH})$, time of wetness (ToW), and salinity can vary significantly over small distances to different microenvironments. The same growth model applies to localized corrosion, however because anodes (pits) are smaller than the surrounding cathodic areas, pitting damage can grow considerably faster than uniform damage.
Table 1. GLCM features

\begin{tabular}{|l|l|}
\hline Name & Equation \\
\hline Contrast & $\sum_{i, j}|i-j|^{2} p(i, j)$ \\
\hline Correlation & $\sum_{i, j}\left(i-\mu_{i}\right)\left(j-\mu_{j}\right) p(i, j) / \sigma_{i} \sigma_{j}$ \\
\hline Energy & $\sum_{i, j} p(i, j)^{2}$ \\
\hline Homogeniety & $\sum_{i, j} p(i, j) /(1+|i-j|)$ \\
\hline
\end{tabular}

Table 2. Morphological features

\begin{tabular}{|l|l|}
\hline Name & Equation \\
\hline Roundness & $4 \pi A / p^{2}$ \\
\hline Solidity & $A /$ ConvexArea \\
\hline Eccentricity & $\sqrt{1-(b / a)^{2}}$ \\
\hline$L_{\text {Major }}$ & $\max (2 a, 2 b)$ \\
\hline$L_{\text {Minor }}$ & $\min (2 a, 2 b)$ \\
\hline
\end{tabular}

Table 3. Surface roughness features

\begin{tabular}{|l|c|l|}
\hline Name & Sym & Equation \\
\hline Max Height & $S_{Z}$ & $S_{P}+S_{V}$ \\
\hline Max Peak Height & $S_{P}$ & $\max (Z(x, y))$ \\
\hline Max Valley Depth & $S_{v}$ & $\min (Z(x, y))$ \\
\hline Mean Height & $S_{a}$ & $\frac{1}{A} \iint|Z(x, y)| d x d y$ \\
\hline RMS Height & $S_{q}$ & $\sqrt{\frac{1}{A} \iint|Z(x, y)|^{2} d x d y}$ \\
\hline Skewness & $S_{s k}$ & $\frac{1}{S_{q}^{3}} \frac{1}{A} \iint Z(x, y)^{3} d x d y$ \\
\hline Kurtosis & $S_{k u}$ & $\frac{1}{S_{q}^{4}} \frac{1}{A} \iint|Z(x, y)|^{4} d x d y$ \\
\hline
\end{tabular}

Table 4. Feature-based Performance Metrics

\begin{tabular}{|l|c|l|}
\hline Name & Sym & Equation \\
\hline Correlation Coef. & $r_{X, Y}$ & $\frac{E\left[\left(x_{i}-\mu_{X}\right)\left(y_{i}-\mu_{Y}\right)\right]}{\sigma_{X} \sigma_{Y}}$ \\
\hline $\begin{array}{l}\text { Fisher Discriminant } \\
\text { Ratio }\end{array}$ & $F D R$ & $\frac{\left(\mu_{x}-\mu_{y}\right)^{2}}{\sigma_{x}^{2}+\sigma_{y}^{2}}$ \\
\hline
\end{tabular}

From Kondo's experiments (Sharland, 1987), pit volume tends to increase linearly, which implies that $d p / d t \propto t^{1 / 3}$, or $b=1 / 3$. Furthermore, the depth-to-radius ratio tends to stay constant around 0.7. Assuming a semi-ellipsoidal pit geometry, the cross-sectional surface area can be expressed as,

$$
A_{\text {measured }}(t)=\pi(1.43 \cdot d(t))^{2}+\text { noise },
$$

where the uncertainty around the mean grows with the timevarying Gumbel distribution,

$$
p(d, t)=\frac{1}{\beta(t)} e^{-\left[\frac{d-\mu(t)}{\beta(t)}+e^{-\left[\frac{d-\mu(t)}{\beta(t)}\right]}\right]},
$$

such that $\mu(t)=a t^{b}$ and $\beta(t)=\beta_{0} t^{b}$ 


\subsubsection{Paris' Law}

The classic Paris fatigue crack growth rate for metals is a power law

$$
\frac{d L}{d N}=C \Delta K^{m}
$$

where $L=$ crack size (length or depth), $N=$ number of cycles (like a usage-based time variable), $\Delta K=K_{\max }-K_{\min }$ is stress intensity factor range, and $C, m$ are empirical parameters associated to a material. Stress intensity factor $K$ measures how "concentrated" stresses are around the crack tip. $K$ is proportional to stress load amplitude, $\sqrt{L}$ (or its reciprocal), and a dimensionless correction for geometry $Y(L)$ (e.g., boundaries). For example, a typical situation is a center crack with remote stress applied uniformly,

$$
\Delta K=\Delta S \sqrt{\pi L} \cdot Y(L)
$$

where $\Delta S=S_{\max }-S_{\min }$ over one cycle. $\Delta K$ may also have a correction for crack closure effect (retarding growth). What Paris says is that for a midrange of $\Delta K$ values, valid only during the crack propagation phase, between crack initiation and fracture, a crack will grow at the rate predicted by the straight line. The higher the applied maximum stress, the faster the path to failure, and a bigger crack size can beget an even faster rate (or can slow down if stress is wedged from the inside so that the crack tip gets farther away from the stress as it grows). Regardless of how fast or slow, a positive rate indicates the specimen will eventually fail, especially since its physical dimensions are finite. For metals, $m$ tends to be between 2 and 4 (e.g. 3.4 steel, 2.85 aluminum alloy), though the range is wider.

\subsection{Real-time Electrochemical Measurements}

Linear polarization resistance (LPR) measurements are used as an electrochemical measurement of mass loss, $m_{\text {loss }}$, in real-time. The relationship between $m_{\text {loss }}$ and polarization resistance for the micro-LPR ( $\mu \mathrm{LPR}$ ) sensors used in this paper are described in detail by (Brown, et al., 2014). The real-time pit volume can be computed by dividing the measured $m_{\text {loss }}$ with the density of the metallic alloy, represented by $\rho$. Assuming a semi-ellipsoidal pit geometry where the radius of the cross sectional area is $0.7 d$, then $d(t)$ can be computed as,

$$
d(t)=0.787 \cdot\left(\frac{m_{\text {loss }}(t)}{\rho N_{p i t s}}\right)^{1 / 3}
$$

where $N_{\text {pit }}$ is the total number of pits over the exposed surface area. The density of AA7075-T6 is $2.81 \mathrm{~g} / \mathrm{cm}^{3}$.

\section{EXPERIMENTAl MATERials AND MethodS}

\subsection{Sample Preparation}

Lap joint samples, shown in Figure 2, were made using two 6" by 3" panels made from AA7075-T6 with a thickness of $1 / 8$ ". These panels were secured together with six polycarbonate fasteners. Before assembly of the lap joint each panel was cleaned with a $35 \mathrm{~min}$ immersion into a constantly stirred solution of $50 \mathrm{~g} / \mathrm{L}$ Turco $4215 \mathrm{NC}-\mathrm{LT}$ at $65^{\circ} \mathrm{C}$. After completing this alkaline cleaning, the panels were rinsed with deionized water and immersed into a $70 \%$ solution of nitric acid solution for $5 \mathrm{~min}$ at $25^{\circ} \mathrm{C}$. The samples were then rinsed again in the deionized water and air-dried. Weights were recorded to the nearest fifth significant figure and the samples were stored in a desiccator. Once the panels were prepared and massed, two $\mu$ LPR sensors were installed between the panels. At this point the six polycarbonate fastners were torqued down evenly to $2 \mathrm{~N} \cdot \mathrm{m}$. After assembling the lap joints, the samples were evenly coated with 2 mils of epoxy-based paint and 2 mils of polyurethane on all exposed surfaces. These coatings were allowed to fully seal over a 24 -hour period at $35^{\circ} \mathrm{C}$ before testing.

\subsection{Accelerated Corrosion Testing of Lap Joints}

Corrosion tests were performed in a cyclic corrosion chamber running the ASTM G85 Annex 5 test. This test consisted of two one-hour steps. The first step involved exposing the samples to a salt fog for a period of one-hour at $25^{\circ} \mathrm{C}$. The electrolyte solution composing the fog was $0.05 \%$ sodium chloride and $0.35 \%$ ammonium sulfate in deionized water. This step was followed by a dry-off step, where the fog was purged from the chamber while the internal environment was heated to $35^{\circ} \mathrm{C}$. Each panel was positioned at a $60^{\circ}$ angle with the flex tape facing downward, as not to allow a direct pathway for condensate to travel into the lap joints. Electrical connections for the $\mu$ LPR sensors were made to a data acquisition recorder positioned outside the chamber by passing extension cables through a bulkhead. Temperature, relative humidity, and $\mu \mathrm{LPR}$ data were acquired at $1 \mathrm{~min}$ intervals.

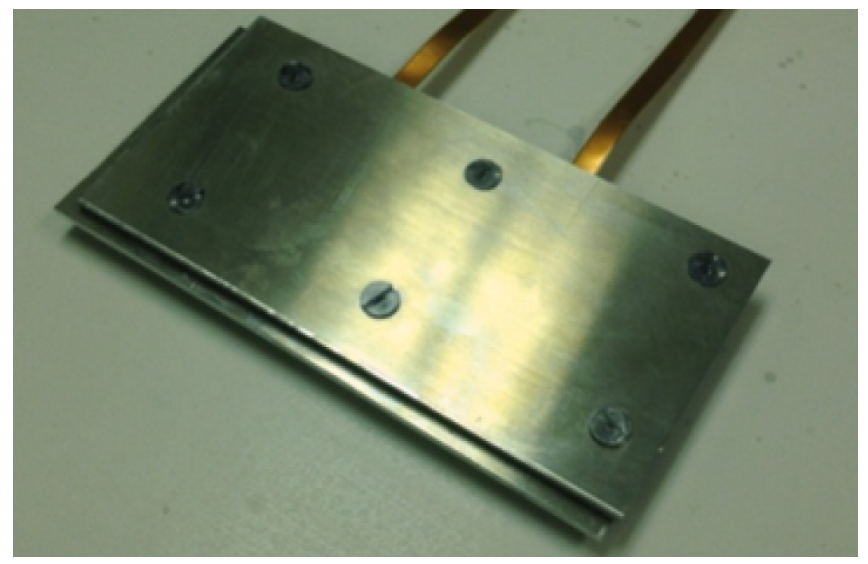

Figure 2. AA7075-T6 lap joint assembly. 


\subsection{Sample cleaning}

After testing, samples were removed from the environmental chamber and disassembled. Following disassembly, the polyurethane and epoxy coatings on the aluminum panels were removed by placing them in a solution of methyl ethyl ketone. After immersion for $30 \mathrm{~min}$ the panels were removed and rinsed with deionized water. These panels were again alkaline cleaned with a 35 min immersion into a constantly stirred solution of $50 \mathrm{~g} / \mathrm{L}$ Turco $4215 \mathrm{NC}-\mathrm{LT}$ at $65^{\circ} \mathrm{C}$. This was followed by a deionized water rinse and immersion into a $90^{\circ} \mathrm{C}$ solution of $4.25 \%$ phosphoric acid containing $20 \mathrm{~g} / \mathrm{L}$ chromium trioxide for $10 \mathrm{~min}$. Following the phosphoric acid treatment, panels were rinsed with deionized water and placed into a $70 \%$ nitric acid solution for $5 \mathrm{~min}$ at $25^{\circ} \mathrm{C}$. Panels were then rinsed with deionized water, dipped in ethanol, and dried with a heat gun. This cleaning process was repeated until mass values for the panels stabilized.

\subsection{Confocal Laser Scanning Microscopy}

Two-dimensional panel images were acquired successively and pre-processed for corrosion image feature extraction. For each panel, 2D microscopic images of size 37 x $37 \mathrm{~mm}$ were taken using LEXT OLS4000, in Error! Reference source not found., with a magnification setting of $108 \mathrm{x}$, and then stitched together to obtain the entire panel image. Error! Reference source not found. depicts the stitched panel microscopic images of Panel 1, 2 and 3 and their corresponding binary images after image pre-processing.

\section{RESULtS}

\subsection{Image Preprocessing}

Preliminary global inspection through the profilometer 3D map scan indicated that the corroded panels were flat without noticeable low-frequency surface irregularities, and thus the surface features can be mostly captured by roughness. Therefore, waviness was omitted for this application. Thus, smoothness and spike removal filters were generally applied at the raw profile measurement from the profilometer and the microscope. Figure 6 (a) and (b) provide the 2D microscopic images of the local pitted panel areas of the same size and magnification in Panel 1 and 2, and Figure 6 (c) and (d) illustrate typical pit cross-sectional profiles from Panel 1 and 2 respectively, with (d) corresponding to the colored line marked in (b). Figure 7 shows a 3D topology image of an area of connected pitting in Panel 2. Table 5 lists the 2D pit profile measurement of the colored lines in both Figure 6 (b) and Figure 7, of which the pit height represents the maximum pit depth.

Except for the 2D pit profile features such as pit width and pit depth, geometric features such as pitting surface area and pit volume can also provide solid measures for local pitting severity, of which pit volume is of importance, due to the

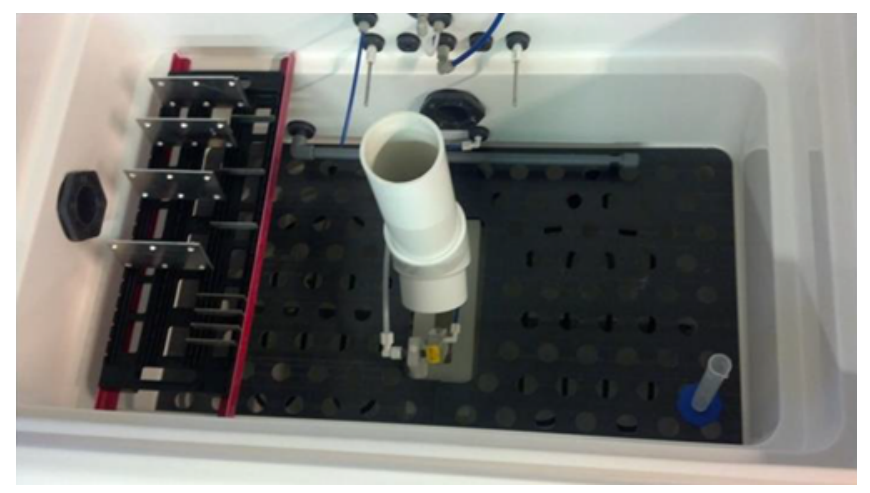

Figure 3. Panels shown in the corrosion chamber prior to the experiment.

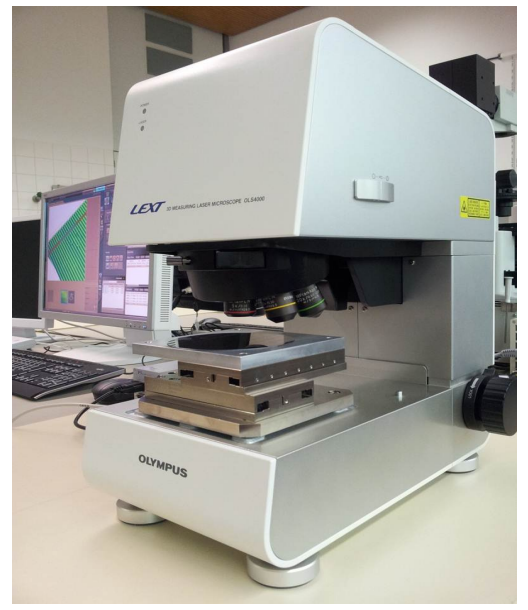

Figure 4. LEXT OLS4000 3D Laser Measuring Microscope.

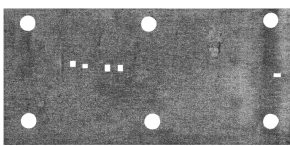

(a)

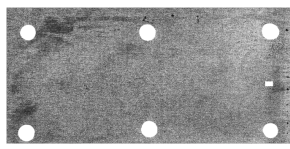

(c)

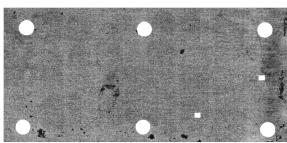

(e)

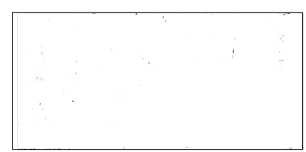

(b)

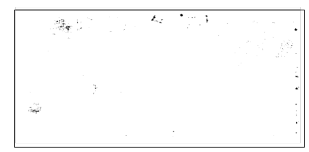

(d)

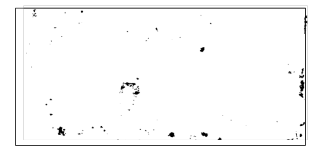

(f)
Figure 5. Whole panel image pre-processing. Left column: intermediary images with rivet holes and marked numbers whitened of (a) Panel 1 with 133-hr corrosion exposure, (c) Panel 2 with 209-hr corrosion exposure, (e) Panel 3 with 286hr corrosion exposure. Right column: binary images after pre-processing of (b) Panel 1, (d) Panel 2, (f) Panel 3.

irregular growth pattern of pitting corrosion. In Figure 7 and Figure 8 , a surface height threshold was manually chosen 
Table 5. Corresponding 2D pit profile measurement of the colored lines in Figure 6 (b) and Figure 7, Panel 2.

\begin{tabular}{|l|l|l|}
\hline Measurement & Figure 6 (b) & Figure 7 \\
\hline Width $(\mu \mathrm{m})$ & 369.432 & 848.483 \\
\hline Height $(\mu \mathrm{m})$ & 3.164 & 19.895 \\
\hline Length $(\mu \mathrm{m})$ & 369.445 & 848.717 \\
\hline
\end{tabular}

Table 6. Corresponding pitting characterization measurements of the area in Figure 7.

\begin{tabular}{|l|l|}
\hline $\begin{array}{l}\text { Cross-sectional Area }\left(\mu \mathrm{m}^{2}\right) \\
\text { (of the red line in Figure 8) }\end{array}$ & $103,366.090$ \\
\hline Surface Area $\left(\mu \mathrm{m}^{2}\right)$ & $192,043.495$ \\
\hline Volume $\left(\mu \mathrm{m}^{3}\right)$ & $1,101,417.185$ \\
\hline
\end{tabular}

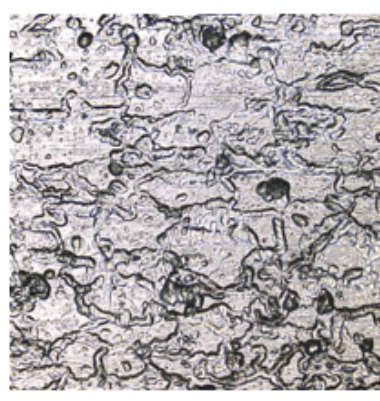

(a)

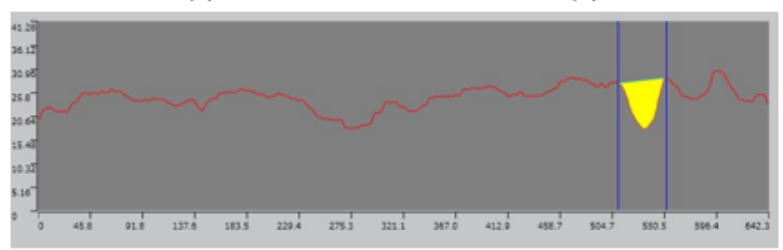

(c)

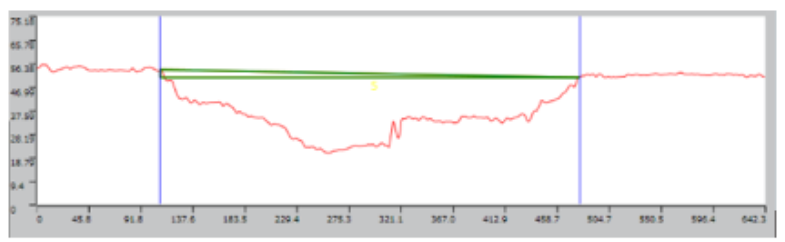

(d)

Figure 6. 2D characterization of pitted panel areas $(642 \times$ $644 \mu \mathrm{m} 2$ ) on the uncoated side of (a) Panel 1, and (b) Panel 2; pit cross-sectional profile measurement (in $\mu \mathrm{m}$ ) of (c) a general pit in Panel 1 (with the highlighted cross-sectional area of $240.43 \mu \mathrm{m} 2$ ), and (d) the colored line in (b), Panel 2.

respectively, in order to calculate the corroded surface area and the underneath pitting volume. The pitting affected surface area was in total of $258,380.787 \mu \mathrm{m}^{2}$, or $3.94 \%$ of the entire examined surface area.

Morphological analysis of the pits in Panel 1 and Panel 2 indicated that, the nucleated pits, as those general non-visible ones in Panel 1, usually took regular morphological forms,

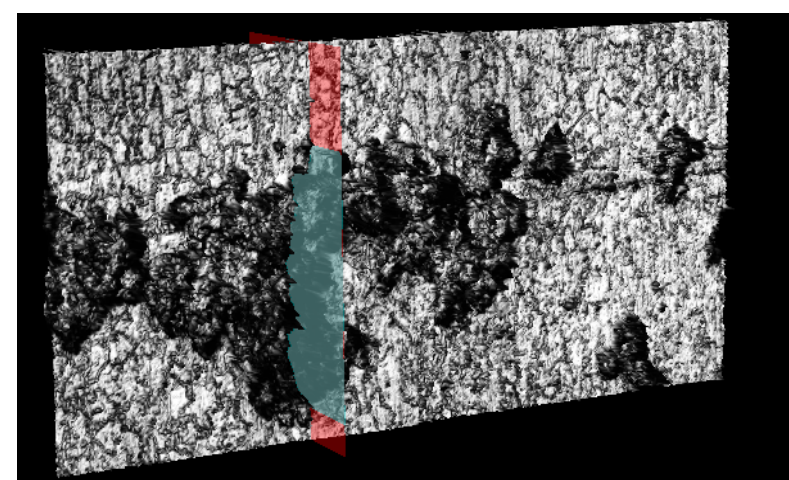

Figure 7. 3D characterization of a pitted panel area $(2561 \times$ $1278 \mu \mathrm{m}^{2}$ ) on the uncoated side of Panel 2, with the corresponding cross-sectional profile measurement as listed in Table 5.
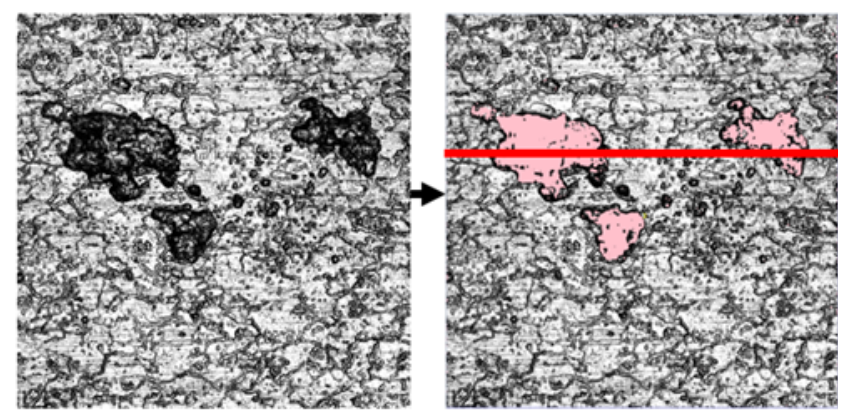

Figure 8. Surface height thresholding procedure to obtain the 3D pitting characterization for a pitted panel area (1278 $\times 1281 \mu \mathrm{m}^{2}$ ) on the uncoated side of Panel 2 .

such as hemispherical, near-hemispherical and near-conical shapes as indicated in Figure 6 (a) and (c). As the corrosion exposure time increased, a few nucleated pits evolved into irregular shapes with the pit dimension increased, as indicated in Figure 6 (b) and (d).

From a side-by-side comparison in Figure 6 (a) and (b), it is noted that, in Panel 2, even though some nucleated pits evolved into bigger and irregular pits, the majority of the pit population were still in a regular shape with similar dimensions as the nucleated pits in Panel 1. Second, as noted from Table 5, a prevalent phenomenon among the large visible pits in Panel 2 and 3 was that, a pit's width was usually significantly larger than its depth, which suggests that the metal dissolution rate was higher at the pit wall than at the pit bottom. In summary, from localized pitting characterization analysis of all three panels, it is concluded that on Panel 1, a number of nucleated pits formed, but generally few visible pits existed; from Panel 1 to 2, as the corrosion exposure time increased from 133 hours to 209 hours, a few visible pits emerged with irregular shapes, with a much larger width than depth; from Panel 2 to Panel 3, as exposure time further increased to 286 hours, a greater number of large visible pits formed, located mostly around panel edges, rivet hole edges, 

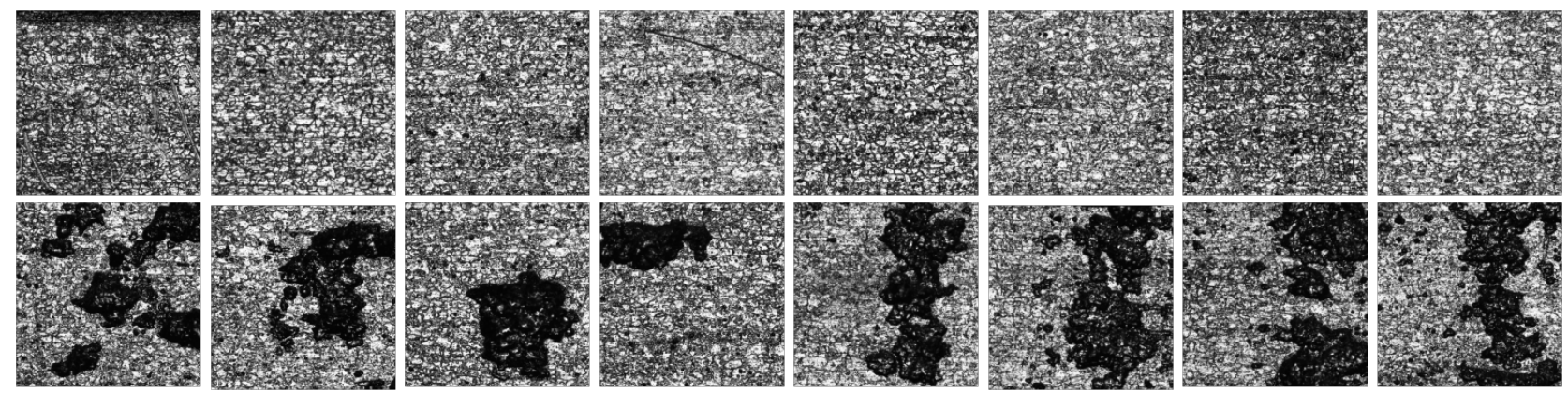

Figure 9. Example corrosion images. Top row: low corrosion. Bottom row: high corrosion.

and surface irregularities. Note that, due to the nature of the accelerated corrosion testing, three panels, instead of one, were exposed to three different corrosion immersion times, respectively. Thus, an individual pit characterization growth cannot be observed in this study. Instead, 3D microscopic characterization studies of a number of random pits was conducted for each panel.

\subsection{Feature Extraction, Selection and Data Mining}

Features extracted from segments of the corrosion images can be used to classify the state of corrosion in the corresponding image segment. Figure 9 shows an example set of corrosion images used for feature extraction. The top row is a set of 8 low corrosion images and the bottom row is a set of 8 high corrosion images. Contrast, correlation, energy, and homogeneity features of the example corrosion images in Figure 9 were calculated and illustrated in Figure 10. The corresponding feature performance was evaluated using FDR as listed in Table 7. This indicates that correlation, energy, and homogeneity are suitable image features for corrosion detection and corrosion state classification, whereas contrast performs poorly.

Figure 11 shows the corroded area percentage of the panels that had corrosion exposure times of 133, 209 and 286 hours. The resulting corroded area percentage feature was highly correlated with the measured panel mass loss as shown in Figure 11. The correlation coefficient $r_{x y}$ of the corroded area percentage and the corresponding measured panel mass loss is 0.9727 .

\subsection{Diagnostic and Prognostic Results}

The diagnostic and prognostic technologies suggested above were applied, in simulation, to the test panel corrosion surfaces. The simulation, conducted in Simulink, utilizes the pitting model in Eq. 6 and the cracking progression model (Paris' Law) in Eq. 9. Figure 12 shows the results of the particle filtering diagnostic routine on the pit depth calculated from $\mu \mathrm{LPR}$ data. The top graph shows the calculated pit depth (green) and the particle filter based estimate of the pit depth (black). The bottom graph shows the detection
Table 7. FDR values of image features.

\begin{tabular}{|c|c|c|c|c|}
\hline Features & Contrast & Correlation & Energy & Homogeneity \\
\hline FDR & 0.9604 & 2.2084 & 95.1962 & 27.3738 \\
\hline
\end{tabular}
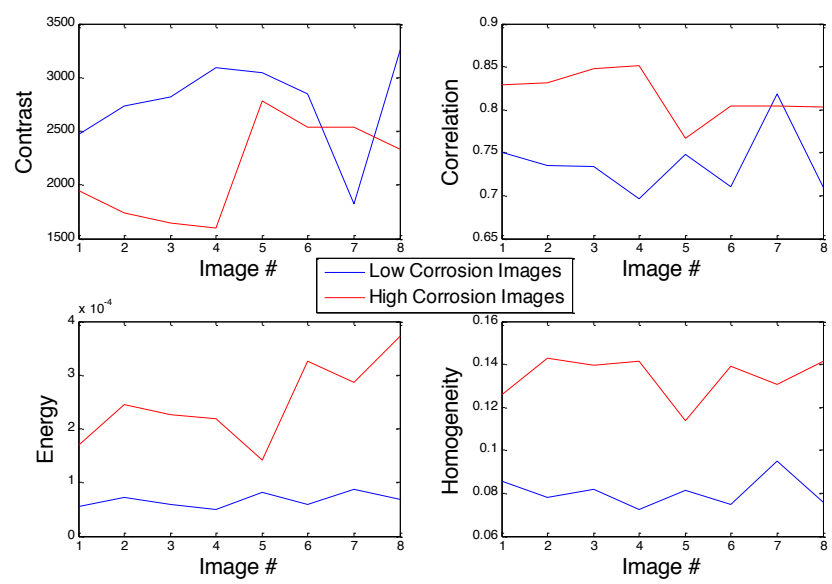

Figure 10. Contrast, Correlation, Energy and Homogeneity features of low and high corrosion images from Figure 9 (image number ascends correspond to the sequence from left to right in each row of Figure 9).
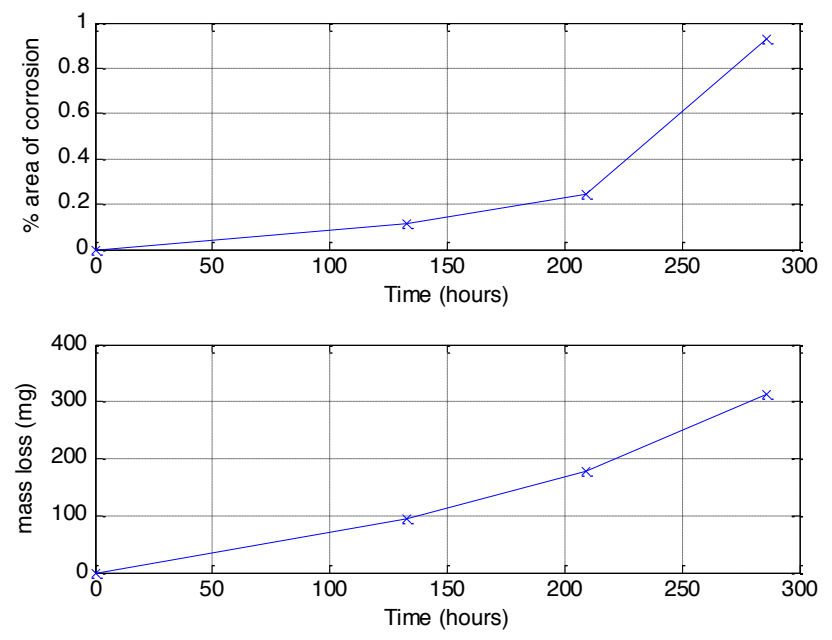

Figure 11. Top: Corroded area percentage over time. Bottom: Measured mass loss (mg) over time. 

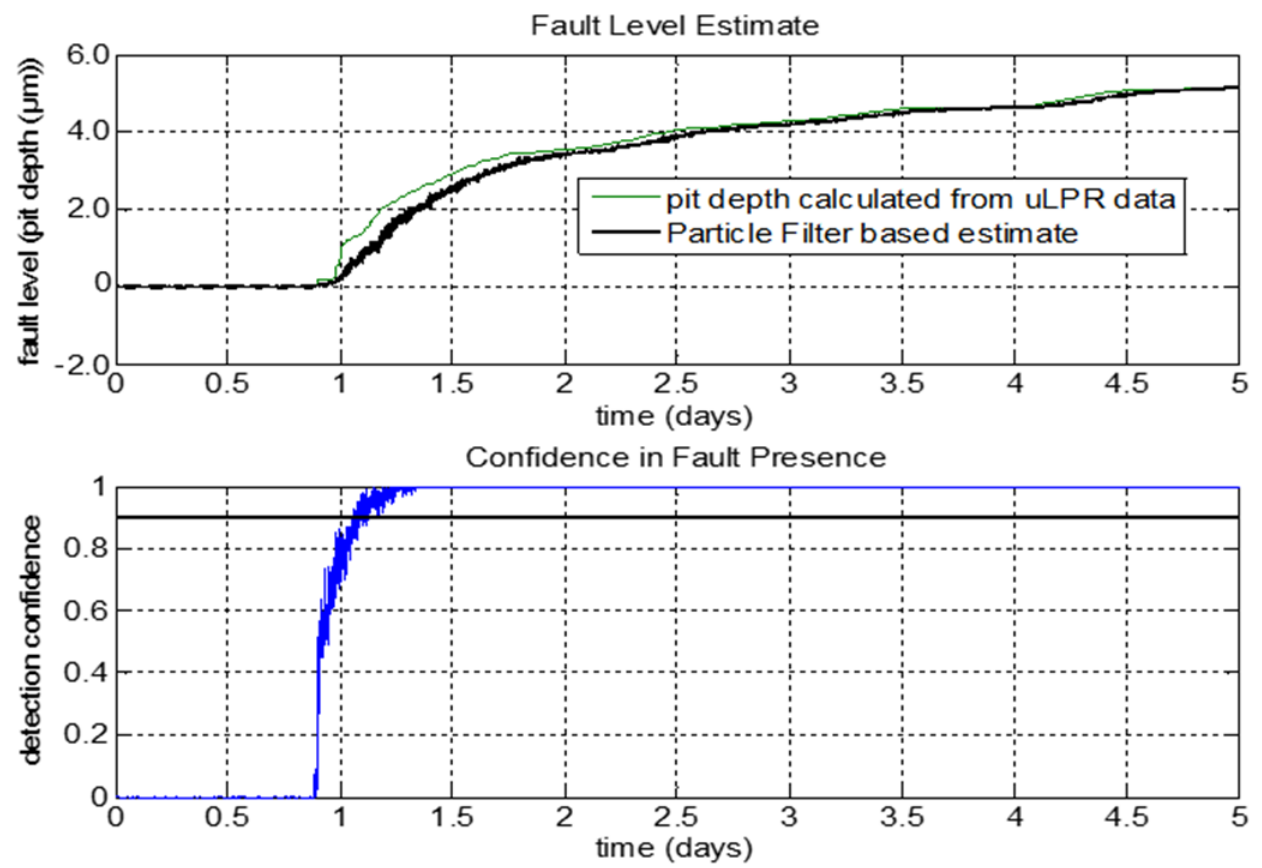

Figure 12. Pit depth from $\mu$ LPR data using the particle filtering methodology.

confidence at each time step. The blue histogram is the baseline distribution and the red one is the current distribution. The black line is the threshold which set the Type I error as $5 \%$. From the figures, it is shown that from time 18 hours, when enough data is collected for generating baseline distribution, to time 36 hours, Type II error is reduced from $78 \%$ to $2 \%$, which means the confidence of abnormal is increased from $22 \%$ to $98 \%$. So at time 36 hours, abnormal is declared.

After the diagnostic routine detects corrosion to a specified confidence level the prognosis routine is run, as shown in Figure 13. The dashed blue line is the measured data of the fault level up to the time of detection. Once corrosion is detected the particles are allowed to progress using the non-linear state model. The failure threshold is user specified and is given as a PDF. The particles progression and the failure threshold PDF is used to calculate the time to failure PDF. The empirical model from Eq. (6) can be employed where $b=1.54$. The following figure is the result of model tuning and prediction. Time is divided into three sections, which are separated by two dashed lines at time $36 \mathrm{~h}$ and $270 \mathrm{~h}$, respectively. Initially, the model parameters are tuned on the basis of available data, as described above. The blue line and green line are upper bound and lower bound of pit depth, respectively. The red dots are the measured pit depth. After the model is tuned, the prediction routine is initiated using only the model. Threshold is shown as the red line, which is $10 \mu \mathrm{m}$. In this case, the predicted remaining useful life is shown in Figure 14 as a PDF. The prognostic results

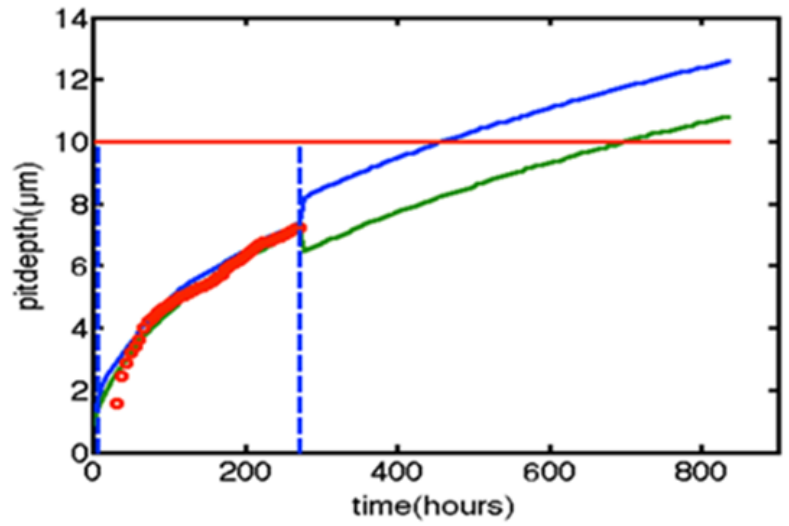

Figure 13. Pit depth prediction scheme.

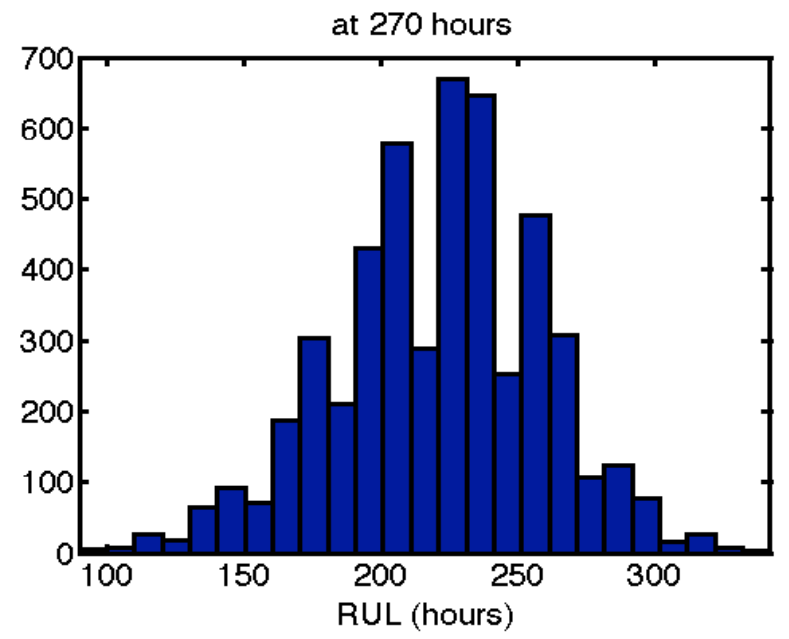

Figure 14. The remaining useful life prediction at 270 hours. 
are matching well-anticipated corrosion predictions in real on-board aircraft applications. The Air Force project reported in this paper has not proceeded to its final stage of on-aircraft testing, data collection, testing and evaluation of diagnostic and prognostic algorithms. Simulation studies and results show the efficacy of the integrated approach to testing, data mining, corrosion initiation and prediction of global and localized corrosion processes.

\section{Conclusions}

This paper introduces a novel and comprehensive framework for corrosion health assessment, integrating robust corrosion testing and monitoring, data mining, corrosion detection, and prediction of corrosion damage growth, with intelligent reasoning paradigms. It is well documented that aircraft corrosion is a major concern that accounts for billions of dollars each year in efforts to detect, quantify, and prevent damage due to corrosion. Although significant advances have been reported over the recent past, there is still an urgent need for new technologies for sensing, data processing, and diagnostic/prognostic algorithm development aimed to provide crucial information to the aircraft community of impending structural failures and a means to mitigate them. The paper addresses the introduction of new methods and tools essential for testing and data processing of corroding panels; such tools become inputs to corrosion diagnostic and prognostic routines. A multitude of challenges remain to be addressed, major among them being the need for accurate and reliable sensing modalities, high fidelity corrosion models and integration architectures for better corrosion detection, prediction and prevention with an ultimate objective to reduce costs and improve the performance of aerospace assets.

\section{ACKNOWLEDGEMENT}

This work was performed with sponsorship from the United States Air Force under Broad Agency Announcement Rapid Innovation Fund contract \# FA8650-12-C-0001.

\section{REFERENCES}

Belanger, P., Cawley, P., \& Simonetti, F. (2010). Guided Wave Diffraction Tomography within the Born Approximation. IEEE Trans UFFC, 57, pp. 14051418.

Brown, D. W., Connolly, R. J., Laskowski, B., Garvan, M., Li, H., Agarwala, V. S., \& Vachtsevanos, G. (2014). A Novel Linear Polarization Resistance Corrosion Sensing Methodology for Aircraft Structure. Annual Conference of the Prognostics and Health Management Society, 5(33).

Brown, D., Darr, D., Morse, J., \& Laskowski, B. (2012). Real-Time Corrosion Monitoring of Aircraft Structures with Prognostic Applications. In Annual Conference of the Prognostics and Health Management Society, 3.
Clark, T. (2009). Guided Wave Health Monitoring of Complex Structures. London, United Kingdom: Imperial College London.

Forsyth, D. S., \& Komorwoski, J. P. (2000). The Role of Data Fusion in NDE for Aging Aircraft. SPIE Aging Aircraft, Airports and Aerospace Haradware IV, 3994, 6.

Frankel, G. S. (1998). Pitting Corrosion of Metals: A Review of the Critical Factors. Journal of the Electrochemical Society, 145(6), pp. 2186-2198.

G102, A. S. (2004). Standard Practice for Calculation of Corrosion Rates and Related Information from Electrochemical Measurements. West Conshohocken, PA: ASTM International.

G59, A. S. (2009). Standard test method for conducting potentiodynamic. West Conshohocken, PA: ASTM International.

Hoeppner, D. W., Chandrasekaran, V., \& Taylor, A. (1999). Review of Pitting Corrosion Fatigue Models. International Committee on Aeronautical Fatigue. Bellevue, WA, USA.

Huang, T. -S., \& Frankel, G. S. (2006). Influence of Grain Structure on Anisotropic Localized Corrosion Kinetics of AA7xxx-T6 Alloys. Corrosion Engineering, Science and Technology, 41(3), pp. 192-199.

Kawai, S., \& Kasai, K. (1985). Considerations of Allowable Stress of Corrosion Fatigue (Focused on the Influence of Pitting). Fatigue Fracture of Engineering Materials $\ \&$ Structures, 8(2), 115-127.

Li, H., Michaels, J. E., Lee, S. J., Michaels, T. E., Thompson, D. O., \& Chimenti, D. E. (2012). Quantification of Surface Wetting in Plate-like Structures via Guided Waves. In AIP Conference Proceedings-American Institute of Physics, 1430(1), 217.

Lindley, T. C., Mcintyre, P., \& Trant, P. J. (1982). FatigueCrack Initiation at Corrosion Pits. Metals Technology, 9(1), 135-142.

López De La Cruz, J., Lindelauf, R., Koene, L., \& Gutiérrez, M. A. (2007, February). Stochastic approach to the spatial analysis of pitting corrosion and pit interaction. Electrochemistry Communications, 9(2), 325-330.

McAdam, G., Newman, P. J., McKenzie, I., Davis, C., \& Hinton, B. R. (2005). Fiber Optic Sensors for Detection of Corrosion within Aircraft. Structural Health Monitoring, 4, 47-56.

Orchard, M., \& Vachtsevanos, G. (2009, June). A Particle Filtering Approach for On-Line Fault Diagnosis and Failure Prognosis," Transactions of the Institute of Measurement and Control. Transactions of the Institute of Measurement and Control, 31(3-4), 221246.

Orchard, M., Vachtsevanos, G., \& Goebel, K. (2011). Machine Learning and Knowledge Discovery for Engineering Systems Health Management. In J. Han 
(Ed.), A Combined Model-Based and Data-Driven Prognostic Approach for Aircraft System Life Management (pp. 363-394). Boca Raton, FL., USA: Chapman and Hall/CRC.

Pereira, M. C., Silva, J. W., Acciari, H. A., Codaro, E. N., \& Hein, L. R. (2012). Morphology Characterization and Kinetics Evaluation of Pitting Corrosion of Commercially Pure Aluminum by Digital Image Analysis. Materials Sciences \& Applications, 3(5), pp. 287-293.

Pidaparti, R. M. (2007). Strucural Corrosion Health Asessment Using Computational Intelligentce Methods. Structural Health Monitoring, 6(3), pp. 245-259.

Rao, K. S., \& Rao, K. P. (2004). Pitting Corrosion of HeatTreatable Aluminum Alloys and Welds: A Review. Transactions of the Indian Institute of Metals, 57(6), pp. 593-610.

Sharland, S. M. (1987). A Review of the Theoretical Modeling of Crevice and Pitting Corrosion. Pergamon Journals Ltd, 27(3), 289-323.

Straub, D. (2004, June). Generic Approaches to Risk Based Inspection Planning for Steel Structures. Zürich: Institute of Structural Engineering, Swiss Federal Institute of Technology.

Szklarska-Smialowska, Z. (1999). Pitting Corrosion of Aluminum. Cossorion Science, 41(9), pp. 17431767.

Wallace, W., \& Hoeppner, D. W. (1985). AGARD Corrosion Handbook Volume I Aircraft Corrosion: Causese and Case Histories. $A G A R D-A G-278,1$.

Wei, R. P., Liao, C. M., \& Gao, M. (1998). A transmission electron microscopy study of 7075-T6 and 2024-T3 aluminum alloys. Metallurgical and Materials Transactions A, 29A, pp. 1153-1163. 